\title{
Erratum to: Sliding Mode Control with Noninvariance to Disturbances in Systems with Nonlinear Objects
}

\author{
G. L. Degtyarev ${ }^{a}$ and A. S. Meshchanov, ${ }^{a, *}$
}

${ }^{a}$ Tupolev Kazan National Research Technical University, ul. Karla Marksa 10, Kazan, 420111 Russia

*e-mail: mas41@list.ru

Received November 9, 2020

DOI: $10.3103 /$ S1068799820030290

The TeX presentation of formulas was updated in HTML file.

The original article can be found online at https://doi.org/10.3103/S1068799820020075. 